\title{
Collective cell migration requires suppression of actomyosin at cell-cell contacts mediated by DDR1 and the cell polarity regulators Par3 and Par6
}

\author{
Cristina Hidalgo-Carcedo ${ }^{1}$, Steven Hooper ${ }^{1}$, Shahid I. Chaudhry ${ }^{1}$, Peter Williamson ${ }^{2}$, Kevin \\ Harrington $^{3}$, Birgit Leitinger ${ }^{4}$, and Erik Sahai ${ }^{1}{ }^{,}$ \\ 1 Tumour Cell Biology Laboratory, Cancer Research UK London Research Institute, 44 Lincoln's \\ Inn Fields, London, WC2A 3PX, UK \\ 2St George's Hospital, Tooting, London, SW17 0QT, UK \\ ${ }^{3}$ Institute of Cancer Research, 237 Fulham Road, London, SW3 6JB, UK \\ ${ }^{4}$ National Heart and Lung Institute, Imperial College London, South Kensington Campus, London, \\ SW7 2AZ, UK
}

\begin{abstract}
Collective cell migration occurs in a range of contexts: cancer cells frequently invade in cohorts while retaining cell-cell junctions. Here we show that collective cancer cell invasion depends on reducing actomyosin contractility at sites of cell-cell contact. When actomyosin is not downregulated at cell-cell contacts migrating cells lose cohesion. We provide a novel molecular mechanism for this down-regulation. Depletion of Discoidin Domain Receptor 1 (DDR1) blocks collective cancer cell invasion in a range of 2D, 3D and 'organotypic' models. DDR1 co-ordinates the Par3/6 cell polarity complex through its C-terminus binding PDZ domains in Par3 and Par6. The DDR1/Par3/6 complex controls the localisation of RhoE to cell-cell contacts where it antagonizes ROCK-driven actomyosin contractility. Depletion of DDR1, Par3, Par6 or RhoE leads to increased actomyosin at cell-cell contacts, a loss of cell-cell cohesion and defective collective cell invasion.
\end{abstract}

\section{Introduction}

Collective cell migration occurs in a range of developmental situations including the morphogenesis of epithelial and endothelial tissues, border cell migration in Drosophila and lateral line migration in zebrafish ${ }^{1},{ }^{2}$. Various pathologies are also associated with cohesive cell migration; for example, many cancers show collective patterns of invasion ${ }^{3},{ }^{4}$. The molecular regulation of this process is not well understood but some principles are becoming apparent. Co-ordinated protease, integrin and RhoA function is required in the leading cells to remodel the extracellular matrix and facilitate the invasion of subsequent cells ${ }^{5}-8$. Effective migration is likely to require co-ordinated regulation of the cytoskeleton of cells moving in the group. Previous work had indicated that myosin function is greater around the edge of collectively invading groups of cancer cells ${ }^{6}$. This increased actomyosin at the edge of the group required Cdc 42 and MRCK. However, neither the significance nor the mechanism of reduced actomyosin within the interior of the group is clear. 
A variety of transmembrane proteins transmit information about the cellular environment to the interior of the cell9-11. These may interact with soluble factors, insoluble extracellular matrix or other cells. Discoidin Domain Receptor 1 (DDR1) is a single membrane spanning protein that interacts with fibrillar collagen and contains an intracellular kinase domain and various motifs for binding adaptor molecules ${ }^{12}{ }^{14}$. DDR1 is highly expressed in epithelial tissues and can be associated with E-cadherin ${ }^{15}$, although the biological significance of this interaction is not clear.

Here we show that collective cancer cell invasion depends on reducing actomyosin contractility at sites of cell-cell contact. Depletion of DDR1 blocks collective cancer cell invasion. DDR1 binds to the cell polarity regulators Par3 and Par6. The DDR1/Par3/6 complex moderates actomyosin activity at cell-cell contacts by controlling the localisation of RhoE. Together these data provide a new model for modulating actomyosin activity at sites of cell-cell contact and thereby enabling collective cancer cell migration.

\section{Results}

\section{Actomyosin organisation in collective invasion}

Many cancer types including squamous cell carcinoma and breast cancer are frequently observed invading collectively ${ }^{3},{ }^{4}$. Here we investigate actomyosin organisation during collective invasion. Myosin Light Chain (MLC) localised around the outside of cell clusters but was reduced at sites of cell-cell contact in A431 squamous cell carcinoma (SCC) cells invading collectively within a 3D matrix (Figure 1a). Similarly, myosin IIa was localised around the outer margins of collectively invading human SCC samples (Supplementary Figure 1). This organisation was also observed in A431 on 2D substrates (Figure 1a). To learn more about the regulation of actomyosin at cell-cell contacts we probed the localisation of the active pS19-MLC in A431 cells following re-plating as single cells. Over the course of 24 hours almost all cells became incorporated in small clusters. In single moving A431 cells, active MLC is localised all around the cell cortex (Supplementary Figure $1 \mathrm{~b}$ left-hand panels). In cases where single cells meet pS19MLC is present at the initial cell contacts (left-mid panels) but starts to be reduced as cell contacts become more extensive (right-mid panels) and is almost absent from well established sites of cell-cell contact after 24 hours (right panels). Thus as single cells aggregate active MLC is maintained around cell edges that are not in contact with other cells but becomes gradually down-regulated at cell-cell contacts.

Timelapse imaging confirmed that A431 cells move in a co-ordinated manner in vitro. Supplementary Figure 2 shows images of A431 cells co-cultured with fibroblasts taken at $36,41.25$ and 47.5 hours post plating. Cell tracking demonstrates the collective nature of this movement and similar direction of motility of adjacent cells (Supplementary Movie 1 and Supplementary Figure 2c). Similar results were obtained in the absence of fibroblasts. To investigate if reduced MLC activity at cell-cell contacts is important for collective migration we globally increased actomyosin activity in A431 cells co-cultured with stromal fibroblasts. A431 cells expressing a conditionally active ROCK1 construct, ROCK1:ER respond to the addition of tamoxifen with uniform and de-regulated activation of ROCK1 driven contractility ( ${ }^{16}$ and data not shown). This de-regulated actomyosin activation leads to a failure in collective cell migration (Supplementary Movie 2). Supplementary Figure 2 shows images taken at similar time intervals to control cells. Tracing of cell paths (Supplementary Figure 2c) clearly show a dramatic lack of co-ordination in the direction of cell movement when actomyosin contractility is de-regulated. Furthermore, breaks within the groups of cells frequently appeared. These data demonstrate that globally elevated actomyosin activity is detrimental to collective cell migration. 


\section{DDR1 regulates actomyosin organisation}

To learn more about the cohesion and actin organisation of A431 cells we investigated the effect of siRNA-mediated depletion of a range of cytoskeletal regulators and adhesion molecules. A broad range of phenotypes were observed including disrupted cell-cell contacts and altered F-actin at cell-cell contacts. Supplementary Figure 3a\&b shows representative images of the phenotypes observed together with the genes that produce the phenotype when depleted. This data was obtained by transfection of pools of siRNA so we cannot exclude off-target effects for all the genes listed. However, we were able to confirm the effect of depleting the following genes using multiple individual siRNA's: paxillin, RhoE, DDR1, Nck2, p190ARhoGAP, Talin, Cdc42, RhoA, p120 catenin, and E-cadherin. Ambiguous results were obtained with Ptk2 and Diaph1 while other hits were not tested (data not shown).

Depletion of DDR1 led to altered actin organisation. Control cells had a single tight band of F-actin at cell-cell contacts whereas DDR1 depleted cells had two diffuse bands of F-actin either side of the contact (Figure 1c). DDR1 depletion also led to myosin IIa and active pS19-MLC being present all around the cell periphery and not just at cell margins away from cell-cell contacts (Figure 1c). The total levels of pS19-MLC or RhoA-GTP were not affected by DDR1 depletion (Supplementary Figure 4a\&b). Altered actomyosin organisation at cell-cell contacts was also observed in MCF10A breast epithelial cells following DDR1 depletion (Supplementary Figure 4d\&e). DDR1 depleted cells were also more spread than controls, which may indicate altered cell-matrix adhesion (Supplementary Figure 4c). Although DDR1 is a collagen receptor ${ }^{13}$, we were unable to observe defects in adhesion to collagen I when DDR1 was depleted (Supplementary Figure 4g).

The actomyosin organisation at cell-cell contacts in cells lacking DDR1 is similar to immature adherens junction in untransformed epithelial cells ${ }^{17}$. Analysis of adherens junctions revealed that they still formed in cells lacking DDR1and that the level of adherens junction components was not altered (Figure 1c and Supplementary Figure 4d\&e). However, cell-cell adhesion in DDR1 depleted cells was partially impaired (Supplementary Figure 4f). These data indicate that DDR1 is required for reducing actomyosin contractility at cell-cell contacts and the formation of more mature adherens junctions.

\section{DDR1 is required for collective cancer cell invasion}

We next investigated the consequence of DDR1 depletion in collective cancer cell migration in a range of assays. Control A431 cells move in cohorts with all cells moving in a similar direction (Figure 1d and Supplementary Movie 3). In contrast, DDR1-depleted cells moved in multiple different directions leading to disruption of the cell grouping (Figure $1 \mathrm{~d}$ and Movies 4\&5). Furthermore, the collective invasion of strands of squamous cell carcinoma cells in both spheroid and organotypic cultures is dramatically reduced by depletion of DDR1 (Figure 1e\&f, siRNA efficiency in SCC12 cells shown in Supplementary Figure 5h). Multiphoton confocal analysis of organotypic cultures confirmed that SCC12 and A431 cells invading collectively in these assays with actomyosin organisation very similar to that shown in Figure 1a (data not shown and reference ${ }^{6}$ ). Together these data demonstrate that DDR1 is required for collective cancer cell invasion in a range of models.

To learn more about how DDR1 was modulating collective cell invasion we attempted to rescue the defects in cells stably depleted for DDR1 by expressing mutant forms of DDR1 resistant to shRNA. Figure 2a shows that wild-type DDR1 expression in adjacent cells restores the F-actin organisation at cell-cell contacts; expression in isolated cells did not rescue F-actin organisation (Figure 2b). The requirement for DDR1 in both neighbouring cells might indicate that it forms 'trans' dimers. However, the extracellular portion of DDR1 
fused to $\mathrm{Fc}^{18}$ was not able to bind to the surface of A431 cells (data not shown). DDR1 kinase-dead (K618A) ${ }^{19}$ or collagen-binding defective (R105A) ${ }^{20}$ mutants were able to restore F-actin organisation at cell-cell contacts (Figure 2b). However, deletion of the entire discoidin domain $(\triangle \mathrm{DS} 1)$ prevented DDR1 from regulating F-actin at cell-cell contacts (Figure 2b). Expression of DDR1 lacking its intracellular domain (ECTM-GFP) led to a similar phenotype as DDR1 depletion even if a residue critical for collagen binding was mutated (Figure 2c). Surprisingly for a molecule implicated in cell-matrix adhesion, DDR1 localised to cell-cell contacts (Figure 2a\&c). Furthermore we found that endogenous DDR1 localised in the apical zone of cell-cell contacts (Figure 2d). The localisation of DDR1 to cell-cell contacts required E-cadherin function (Figure 2e) and was observed in several human cancer biopsies and cell lines (Supplementary Figure 5b and 6 and ref ${ }^{15}$ ). Interestingly, DDR1 was not observed at cell-cell contacts in E-cadherin negative patient samples, cancer cells capable of experimental blood-borne metastasis or following treatment with the combination of pro-metastatic factors EGF and TGF $\beta$ (Supplementary Figure 5b\&c and Supplementary Figure 6b). Our data suggest that binding to a non-collagen ligand at cell-cell contacts by DDR1 co-ordinates the assembly of a protein complex that suppresses actomyosin function and thereby enables collective cancer cell invasion. We sought to identify such a protein complex.

\section{DDR1 interacts with Par3 and Par6}

Sequence analysis of DDR1 revealed a conserved C-terminus with homology to the PDZbinding motifs in claudins 4,5 , and 6 (Figure $3 a)^{21}$. We therefore tried to identify proteins that bound to a 70 amino-acid C-terminal fragment of DDR1 but not a similar fragment lacking the final valine residue, which is required for binding PDZ domains. A candidate approach revealed that the cell polarity regulator Par3 bound to the C-terminus of DDR1 (Figure 3b). We confirmed this interaction by immune-precipitating Par3 with DDR1 (Figure 3ci). Par6, which acts together with Par3 to regulate cell polarity ${ }^{22}$, also coprecipitated with DDR1 (Figure 3cii). DDR1 interacts most strongly with the first PDZ domain of Par3 and the PDZ domain of Par6 (Figure 3d). Immune-fluorescence demonstrated that Par3 and DDR1 co-localise in the apical domain of cell-cell contacts (Figure 3e) and at cell-cell contacts in invasive squamous cell and breast carcinoma (Supplementary Figure 6). Finally, we demonstrated that depletion of DDR1 disrupted the localisation of Par3 (Figure 3f): total Par3 levels were not affected by DDR1 depletion (data not shown). Interestingly, DDR1 localisation was compromised in Par3 depleted cells suggesting interdependence of their sub-cellular distribution (Figure 3g).

\section{Par3 and Par6 are required for collective invasion}

We next investigated if the interaction with Par3 or Par6 could account for the ability of DDR1 to diminish actomyosin contractility at cell-cell contacts. Strikingly, depletion of Par3 or Par6 resulted in very similar phenotypes to depletion of DDR1. Firstly, active pS19 MLC was no longer reduced at cell-cell contacts in Par3 or Par6 depleted cells and F-actin was frequently organised in diffuse bands either side of the cell-cell contact (Figure $4 a \& b$ ). Furthermore, cells depleted for Par3 or Par6 also failed to move in a cohesive manner (Figure 4c) or invade collectively in an organotypic model of squamous cell carcinoma (Figure 4d). Par3 and Par6 have numerous binding partners; including atypical PKC's, Tiam-1 and $\mathrm{Cdc}_{2} 2^{23}-25$. Depletion of PKC 2 and PKC $\zeta$ or Tiam-1 led to a similar phenotype as DDR1 depletion (data not shown). This suggests that a large multi-protein complex may be involved in regulation of collective cell movement by DDR1

\section{Local regulation of actomyosin by DDR1 involves RhoE}

Finally, we wished to determine whether DDR1 and Par3 controlled regulators of actomyosin contractility. During the siRNA screening that led us to DDR1, we noticed that 
depletion of RhoE and p190ARhoGAP also yielded similar phenotypes (shown in detail in Figure 5a\&b, quantification of the phenotype in Supplementary Figure 3c). Furthermore, depletion of RhoE led to a failure in the co-ordinated migration of cell clusters and reduced invasion in 3D (Figures 5c\&d). RhoE and p190ARhoGAP both antagonise Rho-ROCK driven actomyosin contractility. p190ARhoGAP reduces RhoA activity ${ }^{26}$ while RhoE can both directly inhibit ROCK $1{ }^{27}, 28$ and increase the activity of p190ARhoGAP ${ }^{29}$. In addition, we and others have observed that RhoE is found at sites of cell-cell contact (Figure 5e and ${ }^{30}$ ). We therefore asked if DDR1 was controlling the localisation of RhoE and thereby reducing ROCK function at cell-cell contacts. Figures $5 \mathrm{e} \& \mathrm{f}$ show localisation of RhoE-myc expressed in control and DDR1 shRNA A431 clones. RhoE is found in the cell-cell contacts in control cells but is more diffusely distributed in the cytoplasm in the DDR1-depleted clones. Similar results were obtained following Par3 or Par6 depletion (Figure 5f). Total RhoE levels were not affected by DDR1 depletion (data not shown). Importantly, DDR1 could still be observed at cell-cell contacts following RhoE depletion (Figure $5 \mathrm{~g}$ ). Therefore DDR1 controls the localisation of RhoE and not vice-versa. These data predict that the altered actomyosin organisation at cell junctions following DDR1 depletion results from aberrantly high Rho-ROCK function at cell-cell contacts. To test this hypothesis we treated DDR1 depleted cells with ROCK inhibitor and monitored pS19-MLC and F-actin organisation at cell-cell contacts. Figure 5h shows that the elevated pS19-MLC observed at cell-cell contacts in DDR1 depleted cells was completely dependent on ROCK function. Furthermore, the F-actin organisation in DDR1 depleted cells reverted from two diffuse bands to one single band of F-actin following ROCK inhibitor treatment. Similar results were obtained with RhoA siRNA (data not shown). Other aspects of the phenotype of DDR1 depletion cells, such as the increase in spread area, were not dependent of RhoA or ROCK (Figure 5h). These data indicate that the aberrant actomyosin organisation at cell-cell junctions in cells lacking DDR1 results from elevated ROCK-driven actomyosin contractility.

\section{Discussion}

Collective movement requires the coordination of actomyosin organisation between cells. Actomyosin contractility is high around the edge of the cell cluster and low between cells (Figure 6 and ${ }^{6}$ ). At the margin of the group actomyosin is organised in a supra-cellular structure analogous to the 'purse-string' observed in epithelial wound closure ${ }^{31} 33$ (Supplementary Figure $1 \&$ Figure 6). Both the elevated actomyosin observed around the edges of groups of invading cancer cells and 'purse-string' wound closure are dependent on $\mathrm{Cdc} 42^{6},{ }^{32},{ }^{33}$. Force is transmitted between cells through cell-cell contacts near the edge of group. However, if force is applied uniformly around the cell margin the cell junctions become compromised and co-ordination of movement between neighbouring cells fails (Supplementary Figure 2 and Supplementary Movie 2). Consistent with this, contact inhibition of locomotion and cell-cell repulsion are associated with increased Rho-driven actomyosin contraction function following cell-cell contact ${ }^{34},{ }^{35}$. Therefore a mechanism is required to reduce actomyosin contractility at sites of cell-cell contact. DDR1 acts in a new non-collagen binding capacity at cell-cell contacts. The localisation of DDR1 to cell-cell contacts requires E-cadherin ${ }^{15}$. Once localised at cell-cell contacts, DDR1 helps to recruit Par3 and Par6 and these molecules are required for efficient collective invasion. Interestingly, cell polarity regulators are required for optimal migration in 2D scratch/ wound assays ${ }^{36}{ }_{3}$, which have some aspects of collective nature. Moreover, Par3 and Par6 are required for collective migration of border cells in the Drosophila embryo ${ }^{40}$. The DDR1/ Par3/Par6 complex then controls the localisation of RhoE. RhoE may be localised through the intermediary p190ARhoGAP, which can bind both Par6 and RhoE ${ }^{29},{ }^{41}$. Consistent with this, depletion of p190ARhoGAP gave a similar phenotype to DDR1 or Par3/6 depletion (Figure 5a). Both RhoE and p190ARhoGAP can antagonise Rho-ROCK mediated 
regulation of actomyosin ${ }^{42},{ }^{43}$. The Par3-dependent suppression of actomyosin that we observe reciprocates the suppression of Par3 function by the actomyosin regulator ROCK ${ }^{44}$, 45 . It is likely that the reciprocal nature of these negative interactions serves to robustly segregate Par3 and actomyosin. The DDR1-dependent mechanism that we describe most probably acts together with other proteins to reduce Rho-ROCK function at cell-cell contacts, such as p120 catenin-dependent mechanisms ${ }^{46}$. Our analysis has not yet allowed us to determine all the components of DDR1 complexes at cell-cell contacts.

Various regulators of cell polarity become mis-regulated in cancer and this has been linked to increased metastasis ${ }^{47}, 48,49$. We believe that disruption of DDR1-dependent Par3 localisation to cell-cell contacts might be expected to favour blood-borne metastasis. Our data do not exclude a positive role for DDR1 in metastasis as a collagen receptor. Indeed we found that interference with DDR1 function in metastatic MTLn3 cells reduced their ability to colonise lung tissue (data not shown) Therefore, DDR1 expression may not simply correlate with metastatic ability but it is important to consider whether it is acting in cellmatrix or cell-cell adhesion context. In the former context it may promote single cell cancer invasion and processes such as lung colonisation ${ }^{50}$. In the latter context it may only promote more local and lymphatic invasion and hinder haematogenous metastasis. It is likely that DDR1 engages in different molecular complexes depending whether it is involved in cellcell interactions or cell-matrix interactions. For example, our data suggest that DDR1 does not associate with myosin IIa at cell-cell contacts but it has been reported to associate with myosin IIa in other contexts ${ }^{51}$.

In summary, we describe a mechanism is required to reduce actomyosin contractility at sites of cell-cell contact. DDR1 acts in a new non-collagen binding capacity at cell-cell contacts. DDR1 helps to recruit Par3 and Par6 and this complex then controls the localisation of RhoE, which can antagonise Rho-ROCK mediated regulation of actomyosin. Thus, DDR1 functions at cell-cell contacts to keep actomyosin activity at low levels. Without this reduction in actomyosin activity cell cohesion cannot be maintained during collective cell migration.

\section{Supplementary Material}

Refer to Web version on PubMed Central for supplementary material.

\section{References}

1. Friedl P, Hegerfeldt Y, Tusch M. Collective cell migration in morphogenesis and cancer. Int J Dev Biol. 2004; 48:441-449. [PubMed: 15349818]

2. Friedl P, Gilmour D. Collective cell migration in morphogenesis, regeneration and cancer. Nat Rev Mol Cell Biol. 2009; 10:445-457. [PubMed: 19546857]

3. DiCostanzo D, Rosen PP, Gareen I, Franklin S, Lesser M. Prognosis in infiltrating lobular carcinoma. An analysis of "classical" and variant tumors. The American journal of surgical pathology. 1990; 14:12-23. [PubMed: 2153007]

4. Yamamoto E, Kohama G, Sunakawa H, Iwai M, Hiratsuka H. Mode of invasion, bleomycin sensitivity, and clinical course in squamous cell carcinoma of the oral cavity. Cancer. 1983; 51:2175-2180. [PubMed: 6189571]

5. Wolf K, et al. Multi-step pericellular proteolysis controls the transition from individual to collective cancer cell invasion. Nat Cell Biol. 2007; 9:893-904. [PubMed: 17618273]

6. Gaggioli C, et al. Fibroblast-led collective invasion of carcinoma cells with differing roles for RhoGTPases in leading and following cells. Nat Cell Biol. 2007; 9:1392-1400. [PubMed: 18037882] 
7. Hooper S, Gaggioli C, Sahai E. A chemical biology screen reveals a role for Rab21-mediated control of actomyosin contractility in fibroblast-driven cancer invasion. Br J Cancer. 102:392-402. [PubMed: 19953096]

8. Scott RW, et al. LIM kinases are required for invasive path generation by tumor and tumorassociated stromal cells. J Cell Biol. 191:169-185. [PubMed: 20876278]

9. Lemmon MA, Schlessinger J. Cell signaling by receptor tyrosine kinases. Cell. 141:1117-1134. [PubMed: 20602996]

10. Shattil SJ, Kim C, Ginsberg MH. The final steps of integrin activation: the end game. Nat Rev Mol Cell Biol. 11:288-300. [PubMed: 20308986]

11. Gumbiner BM. Regulation of cadherin-mediated adhesion in morphogenesis. Nat Rev Mol Cell Biol. 2005; 6:622-634. [PubMed: 16025097]

12. Johnson JD, Edman JC, Rutter WJ. A receptor tyrosine kinase found in breast carcinoma cells has an extracellular discoidin I-like domain. Proc Natl Acad Sci U S A. 1993; 90:5677-5681. [PubMed: 8390675]

13. Vogel W, Gish GD, Alves F, Pawson T. The discoidin domain receptor tyrosine kinases are activated by collagen. Mol Cell. 1997; 1:13-23. [PubMed: 9659899]

14. Shrivastava A, et al. An orphan receptor tyrosine kinase family whose members serve as nonintegrin collagen receptors. Mol Cell. 1997; 1:25-34. [PubMed: 9659900]

15. Wang CZ, Yeh YC, Tang MJ. DDR1/E-cadherin complex regulates the activation of DDR1 and cell spreading. Am J Physiol Cell Physiol. 2009; 297:C419-429. [PubMed: 19474292]

16. Croft DR, et al. Conditional ROCK activation in vivo induces tumor cell dissemination and angiogenesis. Cancer research. 2004; 64:8994-9001. [PubMed: 15604264]

17. Zhang J, et al. Actin at cell-cell junctions is composed of two dynamic and functional populations. J Cell Sci. 2005; 118:5549-5562. [PubMed: 16291727]

18. Leitinger B. Molecular analysis of collagen binding by the human discoidin domain receptors, DDR1 and DDR2. Identification of collagen binding sites in DDR2. J Biol Chem. 2003; 278:16761-16769. [PubMed: 12611880]

19. Vogel W, et al. Discoidin domain receptor 1 is activated independently of beta(1) integrin. J Biol Chem. 2000; 275:5779-5784. [PubMed: 10681566]

20. Abdulhussein R, McFadden C, Fuentes-Prior P, Vogel WF. Exploring the collagen-binding site of the DDR1 tyrosine kinase receptor. J Biol Chem. 2004; 279:31462-31470. [PubMed: 15136580]

21. Itoh M, et al. Direct binding of three tight junction-associated MAGUKs, ZO-1, ZO-2, and ZO-3, with the COOH termini of claudins. J Cell Biol. 1999; 147:1351-1363. [PubMed: 10601346]

22. Goldstein B, Macara IG. The PAR proteins: fundamental players in animal cell polarization. Dev Cell. 2007; 13:609-622. [PubMed: 17981131]

23. Izumi Y, et al. An atypical PKC directly associates and colocalizes at the epithelial tight junction with ASIP, a mammalian homologue of Caenorhabditis elegans polarity protein PAR-3. J Cell Biol. 1998; 143:95-106. [PubMed: 9763423]

24. Joberty G, Petersen C, Gao L, Macara IG. The cell-polarity protein Par6 links Par3 and atypical protein kinase C to Cdc42. Nat Cell Biol. 2000; 2:531-539. [PubMed: 10934474]

25. Mertens AE, Rygiel TP, Olivo C, van der Kammen R, Collard JG. The Rac activator Tiam1 controls tight junction biogenesis in keratinocytes through binding to and activation of the Par polarity complex. J Cell Biol. 2005; 170:1029-1037. [PubMed: 16186252]

26. Wildenberg GA, et al. p120-catenin and p190RhoGAP regulate cell-cell adhesion by coordinating antagonism between Rac and Rho. Cell. 2006; 127:1027-1039. [PubMed: 17129786]

27. Riento K, Guasch RM, Garg R, Jin B, Ridley AJ. RhoE binds to ROCK I and inhibits downstream signaling. Mol Cell Biol. 2003; 23:4219-4229. [PubMed: 12773565]

28. Pinner S, Sahai E. PDK1 regulates cancer cell motility by antagonising inhibition of ROCK1 by RhoE. Nat Cell Biol. 2008; 10:127-137. [PubMed: 18204440]

29. Wennerberg K, et al. Rnd proteins function as RhoA antagonists by activating p190 RhoGAP. Curr Biol. 2003; 13:1106-1115. [PubMed: 12842009]

30. Foster R, et al. Identification of a novel human Rho protein with unusual properties: GTPase deficiency and in vivo farnesylation. Mol Cell Biol. 1996; 16:2689-2699. [PubMed: 8649376] 
31. Danjo Y, Gipson IK. Actin 'purse string' filaments are anchored by E-cadherin-mediated adherens junctions at the leading edge of the epithelial wound, providing coordinated cell movement. J Cell Sci. 1998; 111(Pt 22):3323-3332. [PubMed: 9788874]

32. Benink HA, Bement WM. Concentric zones of active RhoA and Cdc42 around single cell wounds. J Cell Biol. 2005; 168:429-439. [PubMed: 15684032]

33. Wood W, et al. Wound healing recapitulates morphogenesis in Drosophila embryos. Nat Cell Biol. 2002; 4:907-912. [PubMed: 12402048]

34. Carmona-Fontaine $\mathrm{C}$, et al. Contact inhibition of locomotion in vivo controls neural crest directional migration. Nature. 2008; 456:957-961. [PubMed: 19078960]

35. Sahin M, et al. Eph-dependent tyrosine phosphorylation of ephexin1 modulates growth cone collapse. Neuron. 2005; 46:191-204. [PubMed: 15848799]

36. Dow LE, et al. The tumour-suppressor Scribble dictates cell polarity during directed epithelial migration: regulation of Rho GTPase recruitment to the leading edge. Oncogene. 2007; 26:22722282. [PubMed: 17043654]

37. Etienne-Manneville S, Manneville JB, Nicholls S, Ferenczi MA, Hall A. Cdc42 and Par6-PKCzeta regulate the spatially localized association of Dlg1 and APC to control cell polarization. J Cell Biol. 2005; 170:895-901. [PubMed: 16157700]

38. Osmani N, Vitale N, Borg JP, Etienne-Manneville S. Scrib controls Cdc42 localization and activity to promote cell polarization during astrocyte migration. Curr Biol. 2006; 16:2395-2405. [PubMed: 17081755]

39. Schmoranzer J, et al. Par3 and dynein associate to regulate local microtubule dynamics and centrosome orientation during migration. Curr Biol. 2009; 19:1065-1074. [PubMed: 19540120]

40. Pinheiro EM, Montell DJ. Requirement for Par-6 and Bazooka in Drosophila border cell migration. Development. 2004; 131:5243-5251. [PubMed: 15456726]

41. Zhang H, Macara IG. The PAR-6 polarity protein regulates dendritic spine morphogenesis through p190 RhoGAP and the Rho GTPase. Dev Cell. 2008; 14:216-226. [PubMed: 18267090]

42. Riento K, Villalonga P, Garg R, Ridley A. Function and regulation of RhoE. Biochem Soc Trans. 2005; 33:649-651. [PubMed: 16042565]

43. Peacock JG, et al. The Abl-related gene tyrosine kinase acts through p190RhoGAP to inhibit actomyosin contractility and regulate focal adhesion dynamics upon adhesion to fibronectin. Mol Biol Cell. 2007; 18:3860-3872. [PubMed: 17652459]

44. Nakayama M, et al. Rho-kinase phosphorylates PAR-3 and disrupts PAR complex formation. Dev Cell. 2008; 14:205-215. [PubMed: 18267089]

45. Simoes Sde M, et al. Rho-kinase directs Bazooka/Par-3 planar polarity during Drosophila axis elongation. Dev Cell. 19:377-388. [PubMed: 20833361]

46. Reynolds AB. p120-catenin: Past and present. Biochim Biophys Acta. 2007; 1773:2-7. [PubMed: 17175391]

47. Schimanski CC, et al. Reduced expression of Hugl-1, the human homologue of Drosophila tumour suppressor gene lgl, contributes to progression of colorectal cancer. Oncogene. 2005; 24:31003109. [PubMed: 15735678]

48. Kuphal S, et al. Expression of Hugl-1 is strongly reduced in malignant melanoma. Oncogene. 2006; 25:103-110. [PubMed: 16170365]

49. Gardiol D, Zacchi A, Petrera F, Stanta G, Banks L. Human discs large and scrib are localized at the same regions in colon mucosa and changes in their expression patterns are correlated with loss of tissue architecture during malignant progression. Int J Cancer. 2006; 119:1285-1290. [PubMed: 16619250]

50. Shimada K, et al. Prostate cancer antigen-1 contributes to cell survival and invasion though discoidin receptor 1 in human prostate cancer. Cancer Sci. 2008; 99:39-45. [PubMed: 17970783]

51. Huang Y, Arora P, McCulloch CA, Vogel WF. The collagen receptor DDR1 regulates cell spreading and motility by associating with myosin IIA. J Cell Sci. 2009; 122:1637-1646. [PubMed: 19401332] 
a

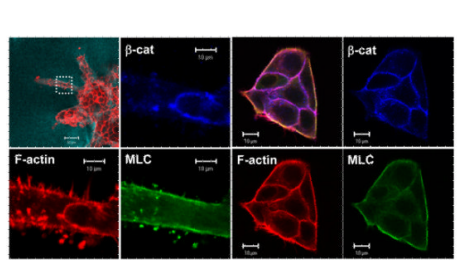

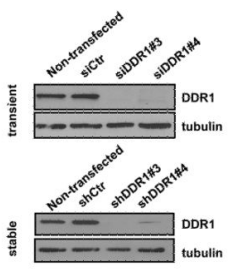

c
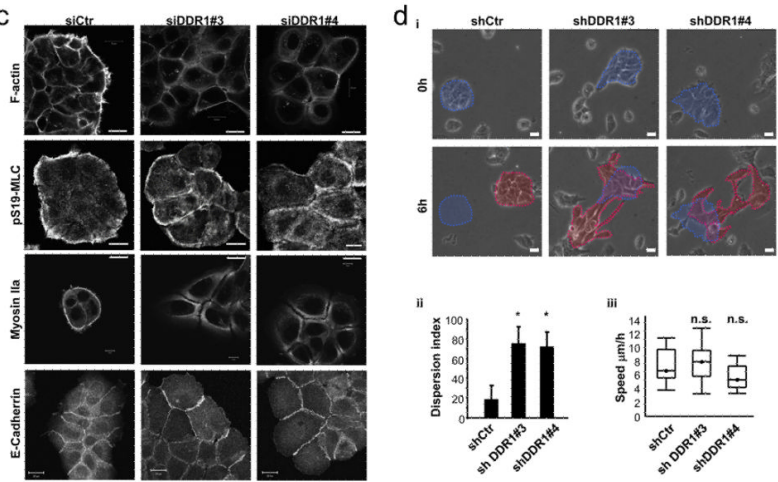

e

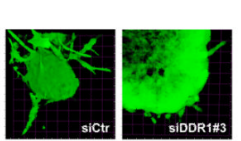

f

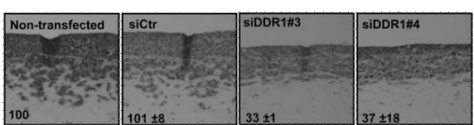

Figure 1. DDR1 is required for collective cell migration

(a) Top-left panel shows A431 SCC cells collectively invading a 3D matrix (F-actin - red, reflectance - cyan). Other left-hand panels show higher magnification of the indicated area. $\beta$-catenin - blue, F-actin - red, GFP-MLC - green. Right-hand panels show A431 cells on a 2D substrate, colours as in left-hand panels. Scale bars are $10 \mu \mathrm{m}$. (b) DDR1 western blots showing efficacy of siRNA (upper panel) and shRNA (lower panel); tubulin is used as loading control. (c) Representative images of F-actin organisation, pS19-MLC, myosin IIa or E-cadherin in A431 cells transfected with control siRNA (left) or with two different siRNA oligonucleotides against DDR1: \#3 (middle) and \#4 (right). Scale bars are $20 \mu \mathrm{m}$. (d) i) Upper panels show snap shots from phase contrast movies of control and DDR1 shRNAtransfected A431 cells moving on collagen gels. Scale bars are $20 \mu \mathrm{m}$. Blue shading shows the position of cell groups at $\mathrm{t}=0$ and red shading shows the position of the same cell groups at $t=6$ hours. ii) and iii) show the cell dispersion index and the average speed of cells within groups, respectively. Data calculated from tracking multiple cell groups from multiple experiments (analysis of 7-10 colonies from three independent experiments). * indicates $\mathrm{p}<0.01$ student's t-test. (e) Panels show 3D reconstruction of 'spheroid' SCC invasion assays in control and DDR1 depleted A431 cells. Grid spacing is 50 $\mathrm{mm}$. (f) Panels show H\&E sections of control or DDR1 siRNA transfected SCC cells in an organotypic assay. The number in the bottom-left corner shows the invasion index of three independent experiments as percentage of the non-transfected cells. 


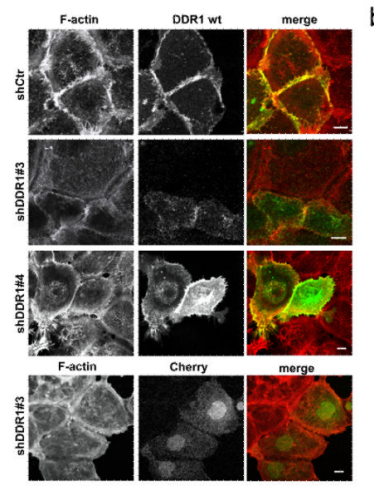

C

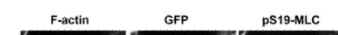

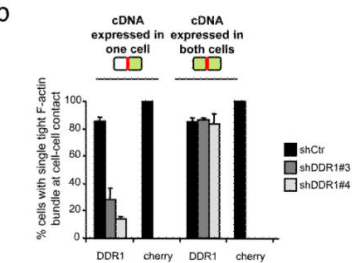

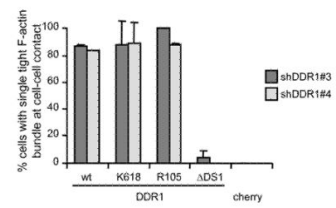

d

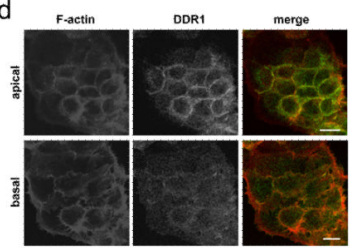

e
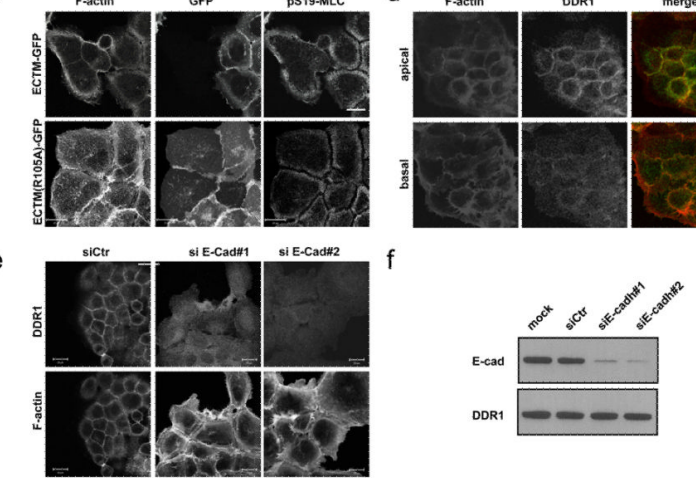

Figure 2. DDR1 does not require kinase activity or collagen binding to regulate acto-myosin at cell contacts

(a) Representative pictures where DDR1 resistant to siRNA oligonucletides \#3\&\#4 was expressed in two DDR1 stable knock down clones (shDDR1\#3 and shDDR1\#4) or control cells (shCtr). F-actin is shown in red and DDR1 in green. The bottom panels show shDDR1\#3 cells transfected with cherry as a control; scale bars are $10 \mu \mathrm{m}$. (b) The upper graph shows the quantification of recovery of normal actin organisation in two DDR1 stable knock down clones (shDDR1\#3 and shDDR1\#4) or control cells (shCtr) transfected with cherry as a control or with a DDR1 construct resistant to siRNA oligonucleotides \#3\&\#4. Left panels show when DDR1 (or cherry) is expressed only in one of the two cells in contact, while right panels show when DDR1 (or cherry) is expressed in both two cells in contact with one another. In the bottom graph is represented the percentage of cells with normal actin organisation in the cell-cell contacts in shDDR1\#3 and shDDR1\#4 cells transfected with cherry as a control or with different DDR1 constructs resistant to siRNA oligonucleotides \#3\&\#4 (wt, K618A, R105A or $\triangle \mathrm{DS} 1$ ). Average of three experiments is shown: 20-30 cells were counted per experiment. (c) F-actin (left) and pS19-MLC organisation in A431 cells transfected with ECTM-GFP (wt in top panels and R105A in bottom panels). In the top panels, cells on the left are non-transfected cells. Scale bars are 20 $\mu \mathrm{m}$. (d) example of DDR1 staining (green) in apical (top panel) and basal (bottom panel) membranes of A431 cells. Scale bars are $20 \mu \mathrm{m}$. (e) F-actin and DDR1 staining is shown in A431 cells transfected with control or E-cadherin siRNA. Scale bar is $20 \mu \mathrm{m}$. (f) Western blot showing E-cadherin and DDR1 levels in A431 cells transfected with control or Ecadherin siRNA. 


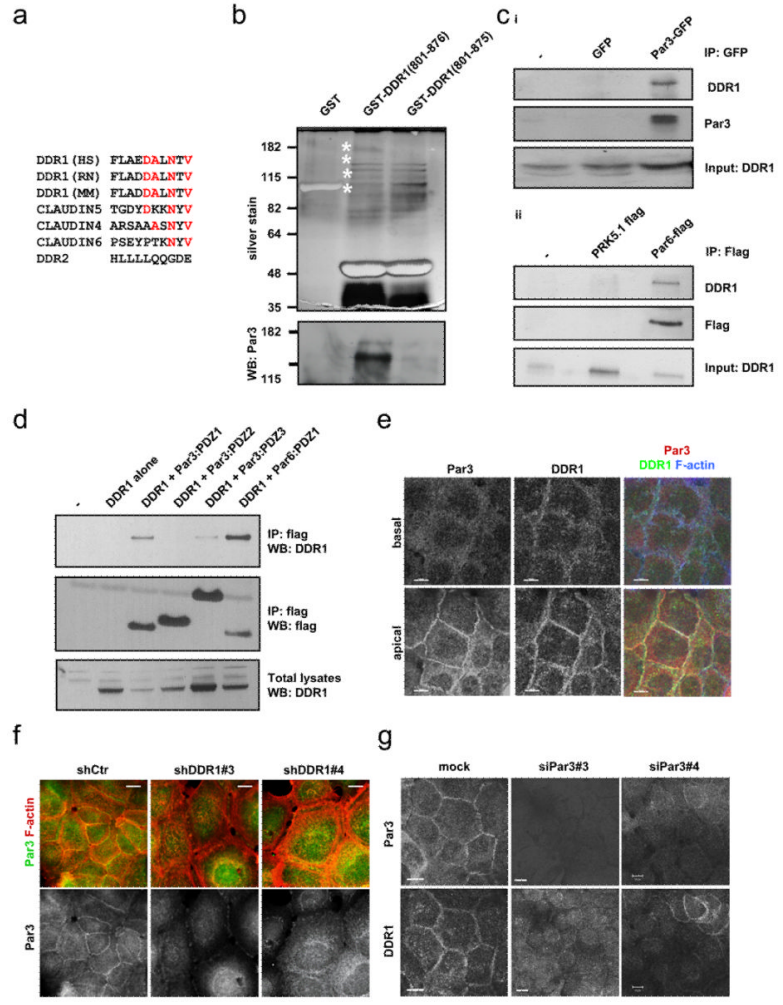

Figure 3. DR1 interacts with Par3 and Par 6

(a) Comparison of the last C-terminal aminoacids of DDR1 in human, rat, mouse, claudin 4, 5 and 6 and DDR2. (b) Negative staining of GST pulldown using GST alone as control (left), DDR1 amino acids 801-876 (middle) and DDR1 amino acids 801-875 (right). Asterisks show bands present only in the 801-876 lane. Bottom panel: Par3 western blot showing Par3 pulled down only by DDR1 801-876. (c) i) Non-transfected (first lane), GFPtransfected (second lane) or Par3-GFP-transfected (third lane) A431 cell lysates were incubated with anti-GFP antibody and the amount of endogenous DDR1 bound was determined by western blot. The Par3 western blot is shown on the middle. The amount of DDR1 in the starting lysates is shown on the bottom. ii) Non-transfected (first lane), empty PRK5.1-transfected (second lane) or Par6-flag-transfected (third lane) A431 cell lysates were incubated with anti-Flag antibody and the amount of endogenous DDR1 bound was determined by western blot. The amount of DDR1 in the starting lysates is shown on the bottom. (d) Flag-tagged PDZ domains of Par3 (third to fifth lanes) and Par6 (sixth lane) were immunoprecipitated from 293 cells also expressing DDR1. Non-transfected cells (first lane) and DDR1 alone (second lane) were used as controls. (e) Representative pictures showing Par3, DDR1 and F-actin staining (blue in merge) in A431 cells in apical membranes. Scale bar is $10 \mu \mathrm{m}$. (f) representative pictures showing actin (red) and Par3 (green) localisation in two clones stably knocked down of DDR1 (shDDR1\#3 or shDDR1\#4) or control A431 cells (shCtr) Scale bars are 10 $\mu \mathrm{m}$. (g) Representative pictures of Par3 (top) and DDR1 (bottom) in A431 cells transfected with control siRNA (left panels) or siRNA against Par3 (right panels). Scale bar is $20 \mu \mathrm{m}$. 
a

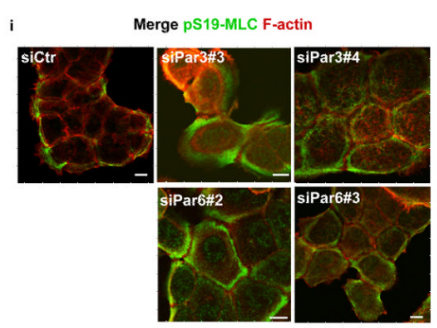

b

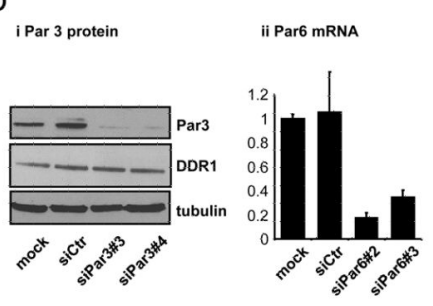

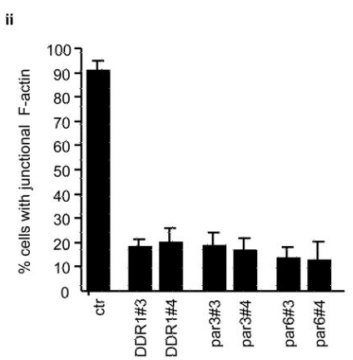

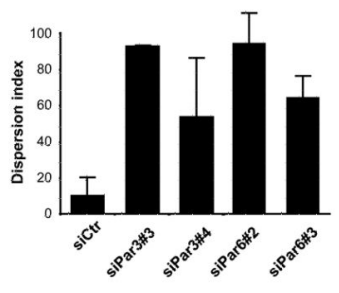

d

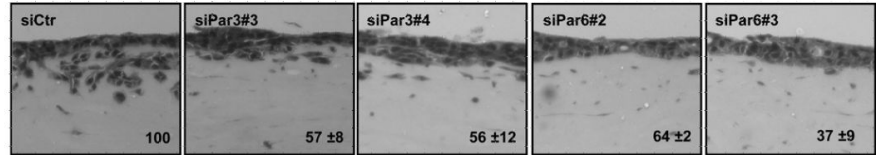

Figure 4. Par3 and Par6 are required for efficient collective invasion

(a) i) Representative images showing pS19-MLC (green) and F-actin (red) localisation in siRNA control, Par3 or Par6 transfections with two different siRNA oligonucleotides. Scale bars are $10 \mu \mathrm{m}$. ii) Quantification of F-actin organization at cell-cell contacts. A431 cells were transfected with the indicated siRNA and 60 hours after transfection were fixed and stained for F-actin. The proportion of cells with tight F-actin at cell-cell contacts was scored ( $\mathrm{n}=2,>200$ cells for each data point). (b) Left-hand panels show Par3 and DDR1 levels in A431 cells transfected with control or Par3 siRNA. $\beta$-tubulin is shown as a loading control. Right-hand panels show QRT-PCR confirmation of Par6 depletion by two siRNA oligonucletides in A431 cells (normalised to GAPDH). (c) Dispersion index of cells moving on collagen gels in control, Par3 or Par6 siRNA transfected A431 cells. Analysis of 7-10 colonies from three independent experiments is shown. * indicates $\mathrm{p}<0.01$ student's t-test. (d) Representative images of H\&E sections of control or Par3 or Par6 siRNA transfected SCC cells in an organotypic assay. The number in the right-bottom corner represents the invasion index after quantification of three independent experiments as percentage of the control cells. 

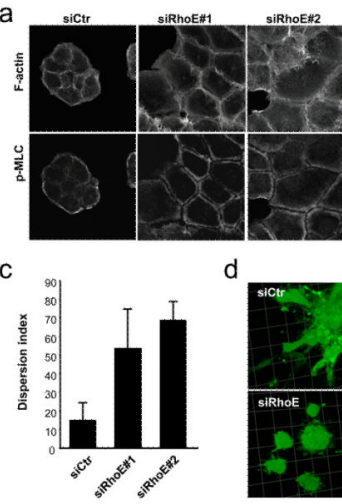

f
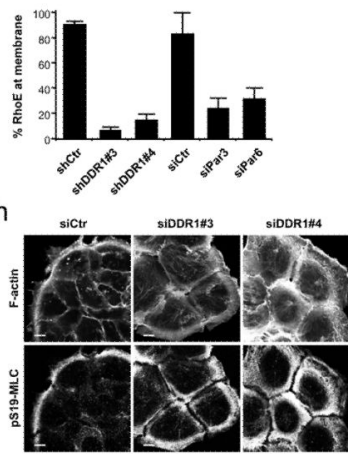

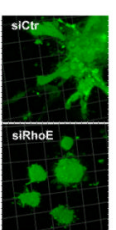

e shctr shDoR1tio
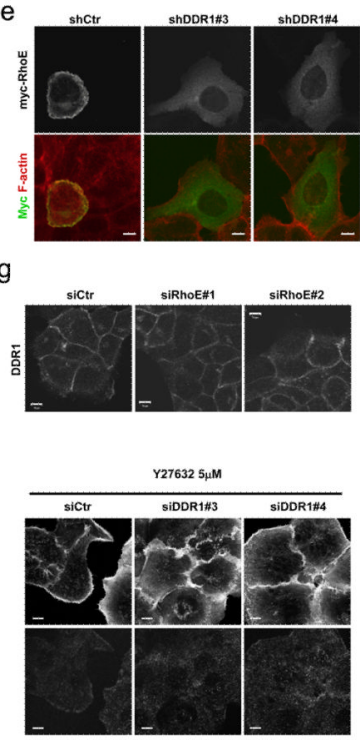

Figure 5. DDR1 controls RhoE localization at cell-cell contacts

(a) Representative images of F-actin organisation or pS19-MLC in A431 cells transfected with control siRNA or with two different siRNA oligonucleotides against RhoE or p190RhoGAP. Scale bars are $10 \mu \mathrm{m}$. (b) RhoE (upper) and p190RhoGAP (lower) western blots showing efficacy of siRNA; $\beta$-tubulin is used as loading control. (c) Dispersion index of cells moving on collagen gels in control or RhoE siRNA transfected A431 cells. Analysis of 7-10 colonies from two independent experiments is shown. * indicates $p<0.01$ student's ttest.. (d) Panels show 3D reconstruction of 'spheroid' SCC invasion assays in control and

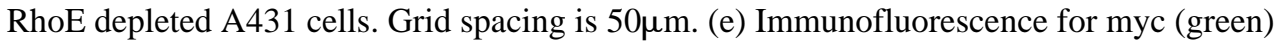
and actin (red) showing RhoE-myc localisation in two DDR1 stable knock down clones (shDDR1\#3 and shDDR1\#4) or control cells (shCtr). Scale bars are $10 \mu \mathrm{m}$. (f) the $\%$ of cells with RhoE localised to the plasma membrane is shown. Average of three experiments is shown: 20-30 cells were counted per experiment. (g) Representative images showing DDR1 localisation in A431 cells transfected with either control or RhoE siRNA. Scale bars are 10 $\mu \mathrm{m}$. (h) Representative images of F-actin and pS19-MLC in control and DDR1 siRNA transfected cells treated with $5 \mu \mathrm{m}$ Y27632 (ROCK inhibitor). Scale bars are $10 \mu \mathrm{m}$. 
Actomyosin organisation in collective cell migration

i) normal situation

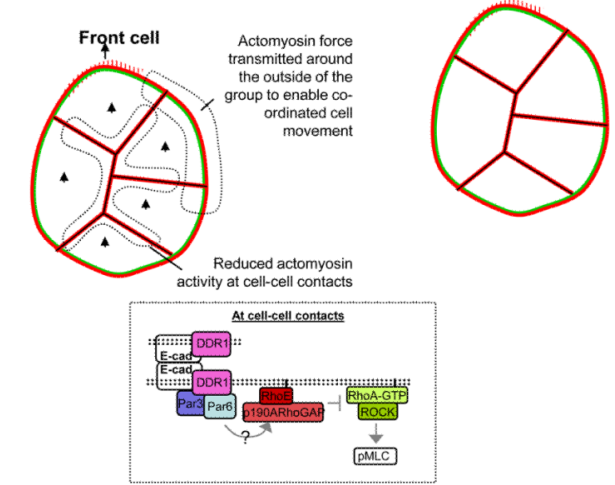

ii) DDR1, Par3, RhoE depleted cells

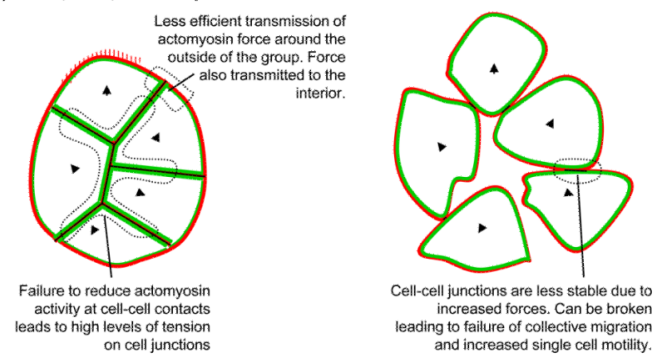

Figure 6.

Schematic model showing the acto-myosin organisation in collective movement in normal conditions (i) or when interfering with DDR1, Par3 or RhoE (ii). 